\title{
Kramers-Heisenberg dispersion formula for scattering of twisted light
}

\author{
Kayn A. Forbes $\oplus^{1, *}$ and A. Salam $\circledast^{2, \dagger}$ \\ ${ }^{1}$ School of Chemistry, University of East Anglia, Norwich NR4 7TJ, United Kingdom \\ ${ }^{2}$ Department of Chemistry, Wake Forest University, Winston-Salem, North Carolina 27109, USA
}

(Received 8 September 2019; published 21 November 2019)

\begin{abstract}
An extremely active research topic of modern optics is studying how light can be engineered to possess forms of structure such as a twisting or a helical phase and the ensuing optical orbital angular momentum (OAM) and its interactions with matter. In such circumstances, the plane-wave description no longer suffices and both paraxial and nonparaxial solutions to the wave equation are desired. Within the framework of molecular QED theory, a general formulation is developed for the scattering of twisted light beams by molecular systems through the Kramers-Heisenberg dispersion formula and ensuing scattering cross section, which takes account of the effects of the phase and intensity structure of twisted light, revealing scattering effects not exhibited by unstructured, plane-wave light. The theory is applicable to linear scattering as well as to nonlinear optical effects for both chiral and nonchiral species, and explicit results are derived for Rayleigh and Raman scattering (including second-order contributions), Rayleigh and Raman optical activity, and their circular-vortex differential scattering analogs. These processes necessitate the inclusion of magnetic-dipole and electric-quadrupole coupling terms, as well as the usual leading electric-dipole interaction term. It is seen that the coupling of electric quadrupole moments to structured light affords a unique sensitivity to the phase properties of the beam, most importantly, its optical $\mathrm{OAM}$, and its inclusion permits the contribution to the scattering cross section proportional to the square of the mixed electric dipole-quadrupole polarizability to be evaluated for which interesting features result. These include its discriminatory behavior arising from circularly polarized input radiation and its dependence on the topological charge, which can also serve to enhance scattering. Also presented are results for a contribution of identical order proportional to the pure electric-dipole and quadrupole polarizabilities.
\end{abstract}

DOI: 10.1103/PhysRevA.100.053413

\section{INTRODUCTION}

The scattering of light by atoms and molecules is a fundamental optical process which alongside absorption and emission accounts for most of the perceivable visual world [1]. The study of scattered light has enabled the determination of structures of biologically and clinically important macromolecules [2,3], nanoparticle characterization [4], and biomedical imaging $[5,6]$, to name but a few examples. The mechanism of light scattering itself is responsible for the gradient force in optical tweezers as well as optical binding, the two most pivotal techniques in optical trapping and manipulation $[7,8]$. Scattering of light in the optical regime is dominated by Rayleigh (elastic) and Raman (inelastic) scattering processes. We may term these scattering phenomena linear, as they both involve the scattering of a single incident photon and as such are linearly dependent on the intensity of the input laser beam. Nonlinear scattering effects include harmonic generation, six-wave mixing, and hyper-Rayleigh and -Raman scattering, all of which fall within the scope of nonlinear optics [9].

In this paper we utilize the theory of molecular quantum electrodynamics (QED) $[10,11]$ to study the linear scattering of light. In QED terminology, both Rayleigh and Raman scat-

\footnotetext{
*k.forbes@uea.ac.uk

†salama@wfu.edu
}

tering are second-order processes involving the annihilation of an incident photon and the creation of a single output photon, where in the former the energies of both photons are identical (hence, elastic), while in the latter the energy of the photons differs by some small amount, this difference being imparted onto the scattering particle, leaving it usually in an excited vibrational state.

The first successful attempt at studying the scattering of light by quantized bound charges was that of Kramers and Heisenberg in 1925, in which by applying the correspondence principle to the classical theory of scattering they derived the now well-known Kramers-Heisenberg dispersion formula, which gives the cross section of scattering by an atom [12]. However this dispersion formula is not fully rigorous in that it cannot explain emission of light quanta, and after further improvements by Born et al. [13], Dirac finally applied the full quantum theory of radiation (and matter) to the problem [14]. Dirac therefore delivered a dispersion formula that can rigorously account for the spontaneous generation (emission) of new modes, vital to understanding the incoherent Raman and Rayleigh scattering effects. This quantum electrodynamical result for the dispersion formula was first derived using the minimal-coupling Hamiltonian, where by invoking the approximation that the spatial variation of the vector potential over the atom or molecule may be neglected, the result can be cast in a form that explicitly contains the electric-dipole transition moments and thus gives the quantum form of the Kramers-Heisenberg dispersion formula that is generally 
quoted. However, by utilizing the multipolar Hamiltonian it has been shown that the quantum form for the dispersion formula may be extended to include all higher-order multipolar contributions to scattering, such as magnetic-dipole and electric-quadrupole terms, in a closed form $[15,16]$.

A very important topic in modern optics that has been subjected to much research effort is structured light $[17,18]$. Beams of laser light can be made to exhibit a whole array of unique structures that entail a plethora of interesting properties. Arguably the most prominent of these structured laser beams is the optical orbital angular momentum (OAM), conveying twisted light structures exhibited by optical vortices such as Laguerre-Gaussian (LG) beams, for example [19]. The OAM from these twisted laser modes stem from the fact that they propagate with a helical-phase structure $\mathrm{e}^{i \ell \phi}$, which indicates the existence of a phase singularity at the center of the beam, and this azimuthal phase dependence provides each photon an OAM of $\ell \hbar$, where $\ell$ is the topological charge or winding number indicating the integer number of twists the light makes within a single wavelength. This remarkable property of laser light is readily observed in the laboratory, inducing forces and torques upon subjected particles, as well as finding a wider range of applications in fields such as freespace communication and information transfer, molecular and atomic optics, and quantum information and entanglement studies [20,21].

Presently, the Kramers-Heisenberg dispersion formula accounts for scattering of unstructured, plane-wave light. The purely theoretical notion of an infinitely extending plane wave is, of course, unphysical, though more often than not it does give adequate results for describing light, particularly when interacting with a material that is much smaller than the incident wavelength [22]. As such, theoretical descriptions of light-matter interactions derived or understood through a plane-wave description of the light may not in general correctly describe an interaction if the light is structured or confined to finite dimensions. Indeed, studies concerned with the scattering of structured light have already discovered such anticipated novel effects [23-30] and applications [31,32]. In this paper we derive the equivalent of the Kramers-Heisenberg dispersion formula for twisted light. We utilize QED methodology to obtain scattering cross sections, taking into account multipolar contributions to scattering, not only from the dominant electric-dipole coupling term but also the magneticdipole and electric-quadrupole interaction terms. Including these higher-order moments is not only justified on account of yielding more general and accurate results, but they both have uniquely important implications in the study of chiral light-matter interactions, as well as the fact that structured light interacts in particularly novel and important ways with the electric quadrupole moment [33-37].

First we give a brief overview of QED theory for twisted laser beams in Sec. II; the Kramers-Heisenberg dispersion formula for twisted light is then derived in Sec. III; we then extract various multipolar contributions to scattering cross sections from our dispersion formula, highlighting agreements with recent studies as well as producing new results in Secs. IV and V; and we then conclude in Sec. VI with a discussion of further applications and future avenues of research.

\section{MOLECULAR QUANTUM ELECTRODYNAMICS AND TWISTED LIGHT}

For applications in optics it is generally more appropriate to formulate molecular QED theory in terms of the PowerZienau-Woolley (PZW) Hamiltonian rather than using the minimal-coupling scheme [10,38]. The PZW Hamiltonian offers distinct advantages that include casting the light-matter interactions in terms of the electric polarization, magnetization, and diamagnetization fields of the material coupling to the electric and magnetic field (and is thus gauge invariant, as it does not involve the electromagnetic potentials). Moreover, in contrast to the minimal-coupling framework, in the PZW formulation there are no electrostatic interactions (an issue Dirac neglected in his minimal-coupling derivation), as all intermolecular interactions are mediated by the transverse electromagnetic field, i.e., are fully retarded. To simplify its use in subsequent applications that depend only upon specific multipole moments, we may express the PZW interaction Hamiltonian in terms of the generally most significant transition moments, such that

$$
\begin{aligned}
H_{\mathrm{int}}= & \sum_{\xi}\left[-\varepsilon_{0}^{-1} \boldsymbol{\mu}(\xi) \cdot \boldsymbol{d}^{\perp}\left(\boldsymbol{R}_{\xi}\right)-\varepsilon_{0}^{-1} Q_{i j}(\xi) \nabla_{j} d_{i}^{\perp}\left(\boldsymbol{R}_{\xi}\right)\right. \\
& \left.-\boldsymbol{m}(\xi) \cdot \boldsymbol{b}\left(\boldsymbol{R}_{\xi}\right)\right],
\end{aligned}
$$

where for a molecule $\xi$ positioned at $\boldsymbol{R}_{\xi}, \boldsymbol{\mu}$ is the electricdipole moment operator, $\boldsymbol{Q}$ is the electric quadrupole operator, and $\boldsymbol{m}$ is the magnetic-dipole moment operator. Summation over repeated subscript indices on the vector and tensor components is implied throughout the paper. The first term in Eq. (1) represents the leading-order electric-dipole (E1) coupling, the second the electric quadrupole (E2) interaction, and the third the magnetic-dipole (M1) coupling, the last two of which are generally smaller than the E1 interaction by about $10^{-3}-10^{-2} ; \boldsymbol{d}^{\perp}\left(\boldsymbol{R}_{\xi}\right)$ is the transverse electric displacement field and $\boldsymbol{b}\left(\boldsymbol{R}_{\xi}\right)$ is the magnetic field. Although it was mentioned previously that the Kramers-Heisenberg dispersion formula for plane waves has been derived in a form that accounts for all multipolar contributions to scattering [15], in this paper we will only concern ourselves with the most significant E1, M1, and E2 contributions, as this will allow us to highlight specific multipolar contributions to phenomena when the dispersion formula is applied. Furthermore, diamagnetic coupling is neglected since it, along with paramagnetic terms, does not contribute to any of the scattering effects to be revealed below.

The electric displacement and magnetic field vacuummode expansions for Laguerre-Gaussian beams, in the paraxial approximation for modes propagating along the specified direction of $z$, emerge as functions of cylindrical coordinates [39]: the off-axis radial distance $r$, axial position $z$, and azimuthal angle $\phi$ :

$$
\begin{aligned}
\boldsymbol{d}^{\perp}(\boldsymbol{r})= & i \sum_{\boldsymbol{k}, \eta, \ell, p}\left(\frac{\hbar c k \varepsilon_{0}}{2 V}\right)^{1 / 2}\left[\boldsymbol{e}_{\ell, p}{ }^{(\eta)}(\boldsymbol{k}) a_{\ell, p}{ }^{(\eta)}(\boldsymbol{k}) f_{\ell, p}(r)\right. \\
& \left.\times e^{(i k z+i \ell \phi)}-\overline{\boldsymbol{e}}_{\ell, p}^{(\eta)}(\boldsymbol{k}) a_{\ell, p}^{\dagger(\eta)}(\boldsymbol{k}) f_{\ell, p}(r) e^{-(i k z+i \ell \phi)}\right],
\end{aligned}
$$




$$
\begin{aligned}
\boldsymbol{b}(\boldsymbol{r})= & i \sum_{\boldsymbol{k}, \eta, \ell, p}\left(\frac{\hbar k}{2 \varepsilon_{0} c V}\right)^{1 / 2}\left[\boldsymbol{b}_{\ell, p}{ }^{(\eta)}(\boldsymbol{k}) a_{\ell, p}{ }^{(\eta)}(\boldsymbol{k}) f_{\ell, p}(r) e^{(i k z+i \ell \phi)}\right. \\
& \left.-\overline{\boldsymbol{b}}_{\ell, p}^{(\eta)}(\boldsymbol{k}) a_{\ell, p}{ }^{\dagger(\eta)}(\boldsymbol{k}) f_{\ell, p}(r) e^{-(i k z+i \ell \phi)}\right]
\end{aligned}
$$

where for a photon of mode $(k, \eta, \ell, p), k$ is the wave-vector magnitude, $\eta$ the polarization, $\ell$ the topological charge, and $p$ the radial order (i.e., the number of intensity rings of the beam); $a_{\ell, p}{ }^{(\eta)}(\boldsymbol{k})$ and $a_{\ell, p}{ }^{\dagger(\eta)}(\boldsymbol{k})$ are the annihilation and creation operators; $V$ is the quantization volume; $\boldsymbol{e}_{\ell, p}{ }^{(\eta)}(\boldsymbol{k})$ and $\boldsymbol{b}_{\ell, p}{ }^{(\eta)}(\boldsymbol{k})$ are the unit electric and magnetic polarization vectors transverse to $\boldsymbol{k}$, such that $\boldsymbol{b}_{\ell, p}{ }^{(\eta)}(\boldsymbol{k})=\hat{\boldsymbol{k}} \times \boldsymbol{e}_{\ell, p}{ }^{(\eta)}(\boldsymbol{k})$; and, for a beam of waist $w_{0}$, the normalized radial distribution function $f_{\ell, p}(r)$ is

$$
f_{\ell, p}(r)=\frac{C_{p}^{|\ell|}}{w_{0}}\left[\frac{\sqrt{2} r}{w_{0}}\right]^{|\ell|} e^{\left(-r^{2} / w_{0}^{2}\right)} L_{p}^{|\ell|}\left(\frac{2 r^{2}}{w_{0}^{2}}\right) .
$$

In Eq. (4), $C_{p}^{|\ell|}$ is a normalization constant and $L_{p}^{|\ell|}$ is the generalized Laguerre polynomial of order $p$. The fundamental annihilation-creation operator commutation relation for twisted light takes the form [40]

$$
\begin{aligned}
{\left[a^{(\eta)}(\boldsymbol{k}), a^{\dagger\left(\eta^{\prime}\right)}\left(\boldsymbol{k}^{\prime}\right)\right]=} & \left(8 \pi^{3} V\right)^{-1} \delta\left(k_{z}-k_{z}^{\prime}\right) \\
& \times \delta\left(k_{r}-k_{r}^{\prime}\right) \delta\left(k_{\phi}-k_{\phi}^{\prime}\right) \delta_{\eta \eta^{\prime}} .
\end{aligned}
$$

The total Hamiltonian for the light-matter system is $H=$ $H_{\mathrm{mol}}+H_{\mathrm{rad}}+H_{\mathrm{int}}$. The sum of the first two terms is generally much larger than the nonrelativistic interactions that occur at optical frequencies in atomic and molecular optics. As such, we may treat the interaction $H_{\text {int }}$ as a small perturbation to the total system and employ the eigenstates of the material and radiation Hamiltonians as basis states for a secular perturbation theory treatment to compute the rates of optical processes such as scattering. We have thus given an overview of the relatively straightforward formulation QED offers for studying and deriving rates for optical processes with twisted light.

\section{KRAMERS-HEISENBERG DISPERSION FORMULA FOR TWISTED LIGHT}

The distinction between real and virtual transitions in QED is pivotal in order to understand second- and higherorder light-matter interactions. The absorption and emission of single photons described in Appendix A with matrix elements respectively given by (A1)-(A3) and (A5)-(A7) require real, energy-conserving transitions to occur in the material. In higher-order effects the annihilation and creation of photons have no such restriction on intermediate states; only in the final radiation and material state must energy of course be conserved. One of the many touted ways to explain this apparent violation of energy conservation in the intermediate steps is through the time-energy uncertainty principle $\Delta E \Delta t \geqslant \frac{1}{2} \hbar$, such that any excited state can be momentarily produced before almost simultaneously being destroyed.

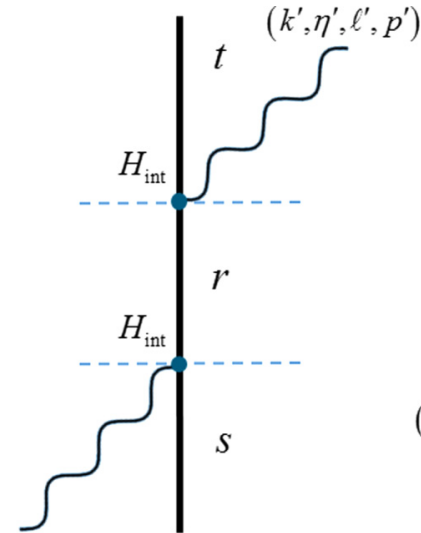

$(k, \eta, \ell, p) \quad(\mathrm{a})$

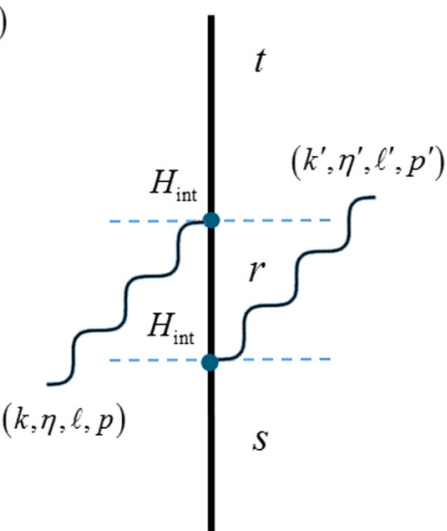

(b)
FIG. 1. Representative time-ordered Feynman graphs for linear scattering of light. Both time orderings (a) and (b) are required in the total calculation for scattering.

Linear scattering is a second-order perturbation process involving the annihilation of an input photon and the creation of an output (scattered) photon. In contrast to single-photon absorption and emission, which involve only real and measurable states, in scattering the material (and light) enters any member of a set of intermediate states $|r\rangle$.

The Feynman diagrams used in diagrammatic timedependent perturbation theory that describe the process of linear scattering are shown in Fig. 1. The matrix element is calculated using second-order perturbation theory via

$$
M_{f i}=-\sum_{r} \frac{\left\langle f\left|H_{\text {int }}\right| r\right\rangle\left\langle r\left|H_{\text {in }}\right| i\right\rangle}{E_{r}-E_{i}} .
$$

First we calculate the contributions to the total matrix element $M_{f i}$ from graph (a) of Fig. 1, $M_{f i}^{a}$, using our first-order matrix elements from Appendix $\mathrm{A}$ for the annihilation and creation of photons by a molecule (A1)-(A3) and (A5)-(A7), respectively. Let the initial state $|i\rangle$ of the total system contain $n$ photons in the mode $(k, \eta, \ell, p)$ and a molecule in a state $|s\rangle$, which after the scattering event leaves the system in a final state of $|f\rangle=\left|t ;(n-1)(k, \eta, \ell, p), 1\left(k^{\prime}, \eta^{\prime}, \ell^{\prime}, p^{\prime}\right)\right\rangle$ :

$$
\begin{aligned}
\left\langle r_{a}\left|H_{\text {int }}\right| i\right\rangle= & -i\left(\frac{n \hbar c k}{2 \varepsilon_{0} V}\right)^{\frac{1}{2}} f_{\ell, p}(r)\left[e _ { j } \left(\mu_{j}^{r s}+Q_{j l}^{r s}\right.\right. \\
& \left.\times\left\{\left[f_{\ell, p}^{-1}(r) \partial_{r} f_{\ell, p}(r)-r^{-1}\right] \hat{r}_{l}+i \ell r^{-1} \hat{\phi}_{l}+i k \hat{z}_{l}\right\}\right) \\
& \left.+c^{-1} b_{j} m_{j}^{r s}\right] e^{i k z} e^{i \ell \phi},
\end{aligned}
$$

and

$$
\begin{aligned}
\left\langle f\left|H_{\text {int }}\right| r_{\mathrm{a}}\right\rangle= & i\left(\frac{\hbar c k^{\prime}}{2 \varepsilon_{0} V}\right)^{\frac{1}{2}} f_{\ell^{\prime}, p^{\prime}}(r) \\
& \times\left[\overline { e } _ { i } ^ { \prime } \left(\mu_{i}^{t r}+Q_{i k}^{t r}\left\{\left[f_{\ell^{\prime}, p^{\prime}}^{-1}(r) \partial_{r} f_{\ell^{\prime}, p^{\prime}}(r)-r^{-1}\right] \hat{r}_{k}\right.\right.\right. \\
& \left.\left.\left.-i \ell^{\prime} r^{-1} \hat{\phi}_{k}-i k^{\prime} \hat{z}_{k}\right\}\right)+c^{-1} \bar{b}^{\prime}{ }_{i} m_{i}^{t r}\right] e^{-i k^{\prime} z} e^{-i \ell^{\prime} \phi},
\end{aligned}
$$


which using (6) thus gives $M_{f i}^{a}$ as

$$
\begin{aligned}
M_{f i}^{a}= & -\sum_{r}\left[n k k^{\prime}\right]^{\frac{1}{2}}\left(\frac{\hbar c}{2 \varepsilon_{0} V}\right) f_{\ell^{\prime}, p^{\prime}}(r) f_{\ell, p}(r) e^{i\left(k-k^{\prime}\right) z} e^{i\left(\ell-\ell^{\prime}\right) \phi}\left(\frac{1}{E_{r s}-\hbar c k}\right) \\
& \times\left[\bar{e}_{i}^{\prime}\left(\mu_{i}^{t r}+Q_{i k}^{t r}\left\{\left[f_{\ell^{\prime}, p^{\prime}}^{-1}(r) \partial_{r} f_{\ell^{\prime}, p^{\prime}}(r)-r^{-1}\right] \hat{r}_{k}-i \ell^{\prime} r^{-1} \hat{\phi}_{k}-i k^{\prime} \hat{z}_{k}\right\}\right)+c^{-1} \bar{b}_{i}^{\prime} m_{i}^{t r}\right] \\
& \times\left[e_{j}\left(\mu_{j}^{r s}+Q_{j l}^{r s}\left\{\left[f_{\ell, p}^{-1}(r) \partial_{r} f_{\ell, p}(r)-r^{-1}\right] \hat{r}_{l}+i \ell r^{-1} \hat{\phi}_{l}+i k \hat{z}_{l}\right\}\right)+c^{-1} b_{j} m_{j}^{r s}\right] .
\end{aligned}
$$

Similarly, the contribution to the matrix element from graph (b) of Fig. 1, $M_{f i}^{b}$, is calculated using

$$
\begin{aligned}
\left\langle r_{b}\left|H_{\text {int }}\right| i\right\rangle= & i\left(\frac{\hbar c k^{\prime}}{2 \varepsilon_{0} V}\right)^{\frac{1}{2}} f_{\ell^{\prime}, p^{\prime}}(r) \\
& \times\left[\bar{e}_{i}^{\prime}\left(\mu_{i}^{r s}+Q_{i k}^{r s}\left\{\left[f_{\ell^{\prime}, p^{\prime}}^{-1}(r) \partial_{r} f_{\ell^{\prime}, p^{\prime}}(r)-r^{-1}\right] \hat{r}_{k}-i \ell^{\prime} r^{-1} \hat{\phi}_{k}-i k^{\prime} \hat{z}_{k}\right\}\right)+c^{-1} \bar{b}_{i}^{\prime} m_{i}^{r s}\right] e^{-i k^{\prime} z} e^{-i \ell^{\prime} \phi}
\end{aligned}
$$

and

$$
\left\langle f\left|H_{\mathrm{int}}\right| r_{b}\right\rangle=-i\left(\frac{n \hbar c k}{2 \varepsilon_{0} V}\right)^{\frac{1}{2}} f_{\ell, p}(r)\left[e_{j}\left(\mu_{j}^{t r}+Q_{j l}^{t r}\left\{\left[f_{\ell, p}^{-1}(r) \partial_{r} f_{\ell, p}(r)-r^{-1}\right] \hat{r}_{l}+i \ell r^{-1} \hat{\phi}_{l}+i k \hat{z}_{l}\right\}\right)+c^{-1} b_{j} m_{j}^{t r}\right] e^{i k z} e^{i \ell \phi},
$$

yielding

$$
\begin{aligned}
M_{f i}^{b}= & -\sum_{r}\left[n k k^{\prime}\right]^{\frac{1}{2}}\left(\frac{\hbar c}{2 \varepsilon_{0} V}\right) f_{\ell^{\prime}, p^{\prime}}(r) f_{\ell, p}(r) e^{i\left(k-k^{\prime}\right) z} e^{i\left(\ell-\ell^{\prime}\right) \phi}\left(\frac{1}{E_{r s}+\hbar c k^{\prime}}\right) \\
& \times\left[e_{j}\left(\mu_{j}^{t r}+Q_{j l}^{t r}\left\{\left[f_{\ell, p}^{-1}(r) \partial_{r} f_{\ell, p}(r)-r^{-1}\right] \hat{r}_{l}+i \ell r^{-1} \hat{\phi}_{l}+i k \hat{z}_{l}\right\}\right)+c^{-1} b_{j} m_{j}^{t r}\right] \\
& \times\left[\bar{e}_{i}^{\prime}\left(\mu_{i}^{r s}+Q_{i k}^{r s}\left\{\left[f_{\ell^{\prime}, p^{\prime}}^{-1}(r) \partial_{r} f_{\ell^{\prime}, p^{\prime}}(r)-r^{-1}\right] \hat{r}_{k}-i \ell^{\prime} r^{-1} \hat{\phi}_{k}-i k^{\prime} \hat{z}_{k}\right\}\right)+c^{-1} \bar{b}_{i}^{\prime} m_{i}^{r s}\right] .
\end{aligned}
$$

The total quantum amplitude for scattering is the sum of (9) and (12):

$$
\begin{aligned}
M_{f i}= & M_{f i}^{a}+M_{f i}^{b}=-\sum_{r}\left[n k k^{\prime}\right]^{\frac{1}{2}}\left(\frac{\hbar c}{2 \varepsilon_{0} V}\right) f_{\ell^{\prime}, p^{\prime}}(r) f_{\ell, p}(r) e^{i\left(k-k^{\prime}\right) z} e^{i\left(\ell-\ell^{\prime}\right) \phi} \\
& \times\left\{\left[\bar{e}_{i}^{\prime}\left(\mu_{i}^{t r}+Q_{i k}^{t r}\left\{\left[f_{\ell^{\prime}, p^{\prime}}^{-1}(r) \partial_{r} f_{\ell^{\prime}, p^{\prime}}(r)-r^{-1}\right] \hat{r}_{k}-i \ell^{\prime} r^{-1} \hat{\phi}_{k}-i k^{\prime} \hat{z}_{k}\right\}\right)+c^{-1} \bar{b}_{i}^{\prime} m_{i}^{t r}\right]\right. \\
& \times\left[e_{j}\left(\mu_{j}^{r s}+Q_{j l}^{r s}\left\{\left[f_{\ell, p}^{-1}(r) \partial_{r} f_{\ell, p}(r)-r^{-1}\right] \hat{r}_{l}+i \ell r^{-1} \hat{\phi}_{l}+i k \hat{z}_{l}\right\}\right)+c^{-1} b_{j} m_{j}^{r s}\right] \frac{1}{E_{r s}-\hbar c k} \\
& +\left[e_{j}\left(\mu_{j}^{t r}+Q_{j l}^{t r}\left\{\left[f_{\ell, p}^{-1}(r) \partial_{r} f_{\ell, p}(r)-r^{-1}\right] \hat{r}_{l}+i \ell r^{-1} \hat{\phi}_{l}+i k \hat{z}_{l}\right\}\right)+c^{-1} b_{j} m_{j}^{t r}\right] \\
& \left.\times\left[\bar{e}_{i}^{\prime}\left(\mu_{i}^{r s}+Q_{i k}^{r s}\left\{\left[f_{\ell^{\prime}, p^{\prime}}^{-1}(r) \partial_{r} f_{\ell^{\prime}, p^{\prime}}(r)-r^{-1}\right] \hat{r}_{k}-i \ell^{\prime} r^{-1} \hat{\phi}_{k}-i k^{\prime} \hat{z}_{k}\right\}\right)+c^{-1} \bar{b}_{i}^{\prime} m_{i}^{r s}\right] \frac{1}{E_{r s}+\hbar c k^{\prime}}\right\} .
\end{aligned}
$$

The resulting Eq. (13) represents the electric-dipole, magnetic-dipole, and electric-quadrupole (E1, M1, and E2, respectively) contributions to the matrix element for the linear scattering of twisted light. Clearly, the E2 interaction is unique [see (A4) in Appendix A] in allowing for the phase structure of a twisted beam to engage in scattering (and light-matter interactions in general), with the purely dipole (E1 and M1) couplings possessing no such sensitivity to the structure of the light.

The rate of scattering follows by invoking Fermi's golden rule rate formula: $\Gamma=2 \pi \hbar^{-1} \rho_{f}\left|M_{f i}\right|^{2}$, where the density of final states for radiation scattered into a cone of solid angle $\mathrm{d} \Omega^{\prime}$ centered around the direction of propagation $\hat{\boldsymbol{k}}^{\prime}$ is $\rho_{f}=$ $k^{\prime 2} \mathrm{~d} \Omega^{\prime} V /(2 \pi)^{3} \hbar c$. Using standard manipulations [10] allows the differential cross section of scattering to be secured as

$$
\frac{d \sigma}{d \Omega^{\prime}}=\frac{k k^{\prime 3}}{16 \pi^{2} \varepsilon_{0}^{2}}\left|M_{f i}\right|^{2}
$$

When $M_{f i}$ is given by Eq. (13), then expression Eq. (14) is the Kramers-Heisenberg dispersion formula for twisted light correct up to E2 coupling.

\section{APPLICATIONS OF THE KRAMERS-HEISENBERG DISPERSION FORMULA FOR TWISTED LIGHT}

In the previous section we produced a generalized quantum amplitude for the scattering of twisted light Eq. (13) and showed how the Kramers-Heisenberg dispersion formula for twisted light Eq. (14) follows in a simple manner. Our result is particularly general and applicable to a plethora of scattering scenarios. First, it applies to both Rayleigh and Raman scattering in nonforward directions and also to Raman scattering in the forward direction; individual multipolar contributions may be extracted, including pure and interference terms; and importantly, while both the input and scattered light may possess 
the Laguerre-Gaussian beam structure and the corresponding optical OAM, neither necessarily have to.

It is worth drawing attention to some of the more intricate details of these features of the dispersion formula and of the scattering of twisted photons. Any Kramers-Heisenberg dispersion formula cannot account for the process of forward Rayleigh scattering, where for the total light-matter system $|i\rangle=|f\rangle$, as the physical observable of this phenomenon is a potential energy rather than an optical rate. Indeed, it is the mechanism of forward Rayleigh scattering that accounts for the well-known gradient force used in optical trapping and tweezer techniques [41].

For an input twisted photon, it is important to note that we cannot make any firm statements about the structure of the scattered light for any directions other than that traveling (forward) along the $z$ axis; the structure of the incident beam, and that of the individual photons, is in general lost upon a nonforward scattering event, unless, of course, the scattered light is resolved for a specific $\ell^{\prime}$. Only those photons scattered in the forward direction have the capability of conveying and maintaining the fidelity of the beam structure, as well as actually reaching a detector [42]. Nonforward Rayleigh scattering of structured light therefore has important consequences in the field of free-space communications, particularly at low levels of intensity, where the optical OAM of structured beams is multiplexed to engender large quantities of information transfer [43-45].

We can use our generalized dispersion formula to calculate the rate of this destructive nonforward scattering. The most straightforward way to model this destructive nonforward scattering is through the incident $n$ photons in mode $(k, \eta, \ell, p)$, to be scattered into a generic unoccupied mode, not in any way specifically structured, i.e., $\left(\boldsymbol{k}^{\prime}, \eta^{\prime}\right)$ and where $\ell^{\prime}=0$ and $p^{\prime}=0$; in such a case the matrix element Eq. (13) then takes the form

$$
\begin{aligned}
M_{f i}= & M_{f i}^{a}+M_{f i}^{b}=-\sum_{r}\left[n k k^{\prime}\right]^{\frac{1}{2}}\left(\frac{\hbar c}{2 \varepsilon_{0} V}\right) f_{\ell, p}(r) e^{i k z} e^{i \ell \phi} e^{-i k^{\prime} \cdot R}\left\{\left[\bar{e}_{i}^{\prime}\left(\mu_{i}^{t r}-Q_{i k}^{t r} i k^{\prime} \hat{k}_{k}^{\prime}\right)+c^{-1} \bar{b}_{i}^{\prime} m_{i}^{t r}\right]\right. \\
& \times\left[e_{j}\left(\mu_{j}^{r s}+Q_{j l}^{r s}\left\{\left[f_{\ell, p}^{-1}(r) \partial_{r} f_{\ell, p}(r)-r^{-1}\right] \hat{r}_{l}+i \ell r^{-1} \hat{\phi}_{l}+i k \hat{z}_{l}\right\}\right)+c^{-1} b_{j} m_{j}^{r s}\right] \frac{1}{E_{r s}-\hbar c k} \\
& +\left[e_{j}\left(\mu_{j}^{t r}+Q_{j l}^{t r}\left\{\left[f_{\ell, p}^{-1}(r) \partial_{r} f_{\ell, p}(r)-r^{-1}\right] \hat{r}_{l}+i \ell r^{-1} \hat{\phi}_{l}+i k \hat{z}_{l}\right\}\right)+c^{-1} b_{j} m_{j}^{t r}\right] \\
& \left.\times\left[\bar{e}_{i}^{\prime}\left(\mu_{i}^{r s}-Q_{i k}^{r s} i k^{\prime} \hat{k}_{k}^{\prime}\right)+c^{-1} \bar{b}_{i}^{\prime} m_{i}^{r s}\right] \frac{1}{E_{r s}+\hbar c k^{\prime}}\right\} .
\end{aligned}
$$

The most important contribution to scattering will generally come from the electric-dipole interactions with the field, and extracting the relevant terms gives the corresponding Kramers-Heisenberg dispersion formula for twisted light as

$$
\frac{d \sigma}{d \Omega^{\prime}}=\frac{k k^{\prime 3}}{16 \pi^{2} \varepsilon_{0}^{2}}\left|f_{\ell, p}(r) \bar{e}_{i}^{\prime} e_{j} \sum_{r}\left[\frac{\mu_{i}^{t r} \mu_{j}^{r s}}{E_{r s}-\hbar c k}+\frac{\mu_{j}^{t r} \mu_{i}^{r s}}{E_{r s}+\hbar c k^{\prime}}\right]\right|^{2},
$$

where the electric-dipole polarizability tensor is defined as

$$
\alpha_{i j}^{t s}\left(\omega,-\omega^{\prime}\right)=\sum_{r}\left[\frac{\mu_{i}^{t r} \mu_{j}^{r s}}{E_{r s}-\hbar c k}+\frac{\mu_{j}^{t r} \mu_{i}^{r s}}{E_{r s}+\hbar c k^{\prime}}\right] .
$$

Up to this point we have not specifically stated whether we are looking at Rayleigh or Raman scattering. The results are however more strictly applicable directly to Rayleigh scattering, with the Raman scattering being easily accounted for by making suitable modifications. These changes are well known and can be found in the literature $[10,46]$, but it is worth outlining them here, particularly as in the next section we will be concerned with optical activity, where the Raman form of optical activity is much more important than the Rayleigh form [47]. The same basic matrix elements and corresponding dispersion formulas derived throughout this article apply to Raman scattering (and Raman optical activity) but with the molecular-polarizability tensors replaced by corresponding vibrational Raman transition tensors. These tensors describe transitions between the initial vibrational state $|v\rangle$ and the final vibrational state $\left|v^{\prime}\right\rangle$, and so, for example, Eq. (17) is replaced by $\left\langle v^{\prime}\left|\alpha_{i j}(Q)\right| v\right\rangle$, where $\alpha_{i j}(Q)$ is the effective polarizability that depends parametrically on the normal vibrational coordi- nates $Q$. This effective polarizability $\alpha_{i j}(Q)$ may be expanded in a Taylor series about the equilibrium position $Q_{\mathrm{e}}$, where the leading term is

$$
\alpha_{i j}^{t s}\left(\omega,-\omega^{\prime}\right)=\left.\frac{\partial \alpha_{i j}(Q)}{\partial Q}\right|_{Q_{\mathrm{e}}}\left\langle v^{\prime}\left|Q-Q_{\mathrm{e}}\right| v\right\rangle .
$$

The Raman intensity is therefore determined by the variation of the polarizability tensor with a normal coordinate of vibration.

\section{ROLE OF HIGHER-ORDER MULTIPOLAR SCATTERING OF TWISTED LIGHT}

An important consequence of including the higher-order M1 and E2 multipolar contributions to scattering, as we have done in our dispersion formula, is that we can study chiroptical phenomena [48]. The interferences of E1 with both M1 and E2 have long been understood as being responsible for 

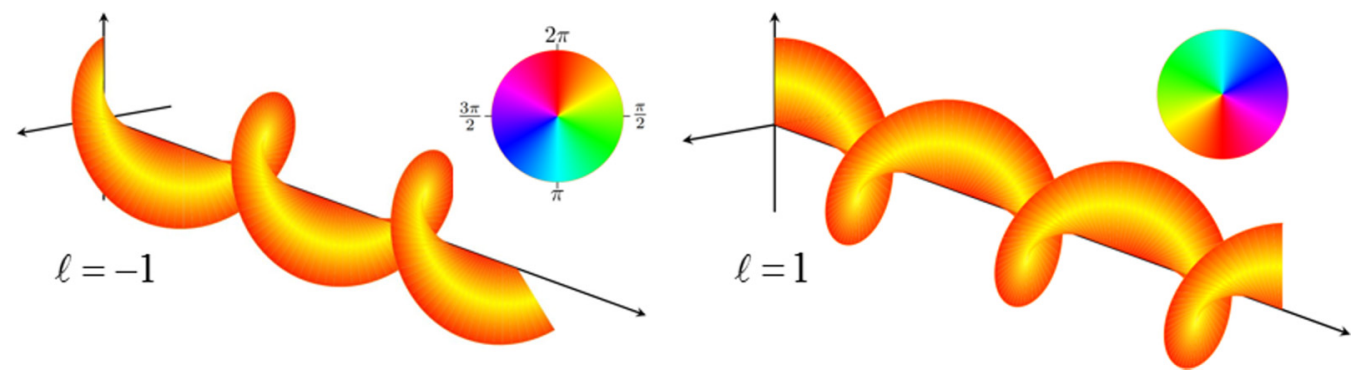

FIG. 2. Twisted light handedness (corresponding azimuthal phase inset) for beams of topological charge $|\ell|=1$. Optical vortices are inherently chiral, screwing to the left for $\ell>0$ and to the right for $\ell<0$ (from the point of view of the source).

the optical activity exhibited by chiral molecules in processes such as optical rotation, circular dichroism, and the differential scattering of circularly polarized light in Rayleigh and Raman optical activity [10,46,47]. Herein we are explicitly interested in Rayleigh and Raman optical activity as they are scattering phenomena. The discriminatory mechanism in these interactions stems from the interferences between the pure electric-dipole polarizability tensor Eq. (17) and the mixed electric-dipole-magnetic-dipole " $G$ " and electricdipole-electric-quadrupole " $A$ " optical activity polarizability tensors. These interferences manifest as " $\alpha G$ " and " $\alpha A$ " couplings and are exhibited only by chiral molecules.

While we may easily extract $\alpha G$ coupling terms from the dispersion formula and therefore derive their corresponding rates to Rayleigh and Raman optical activity, it has recently been established that they show no unique characteristics or dependencies linked to the phase structure of twisted light [49,50]. This is because $\alpha G$ couplings arise from purely dipole interactions (E1 and M1) [51]. However, of more interest to us is that E2 interactions do engage the phase structure of twisted light in significant ways [33], in particular, they give rise to chiroptical effects depending on whether the light twists clockwise or counterclockwise (see Fig. 2) through $\alpha A$ couplings. Indeed, the question of whether the optical OAM of twisted light through the sign of $\ell$ could engage in chiroptical interactions in a similar way to which the spin angular momentum (SAM) of light does through circular polarizations has been an important issue in modern chiral optics. While the original studies concerned with dipole interactions with the field failed to discover any such mechanism for molecules [51], recent studies that included quadrupole interactions discovered that the sign of $\ell$ can play a significant role in discriminatory interactions such as circular-vortex dichroism [52,53] and circular-vortex differential scattering (CVDS) [49,50]. The latter of these, which is a form of Rayleigh and Raman optical activity, should therefore be readily extracted from our dispersion formula for twisted light.

To do so, we again assume incident $n$ photons in the mode $(k, \eta, \ell, p)$ being scattered into a mode $\left(\boldsymbol{k}^{\prime}, \eta^{\prime}\right)$ by a chiral molecule, and as discussed, we need to retain only the E1 and E2 contributions to highlight effects due to the helical-phase structure:

$$
\begin{aligned}
M_{f i}= & -\sum_{r}\left[n k k^{\prime}\right]^{\frac{1}{2}}\left(\frac{\hbar c}{2 \varepsilon_{0} V}\right) f_{\ell, p}(r) e^{i k z} e^{i \ell \phi} e^{-i k^{\prime} \cdot R_{e_{i}^{\prime}}^{\prime} e_{j}} \\
& \times\left\{\left[\left(\mu_{i}^{t r} \mu_{j}^{r s}-\mu_{j}^{r s} Q_{i k}^{t r} i k^{\prime} \hat{k}_{k}^{\prime}+\mu_{i}^{t r} Q_{j l}^{r s}\left\{\left[f_{\ell, p}^{-1}(r) \partial_{r} f_{\ell, p}(r)-r^{-1}\right] \hat{r}_{l}+i \ell r^{-1} \hat{\phi}_{l}+i k \hat{z}_{l}\right\}\right)\right] \frac{1}{E_{r s}-\hbar c k}\right. \\
& \left.+\left[\left(\mu_{j}^{t r} \mu_{i}^{r s}-\mu_{j}^{t r} Q_{i k}^{r s} i k^{\prime} \hat{k}_{k}^{\prime}+\mu_{i}^{r s} Q_{j l}^{t r}\left\{\left[f_{\ell, p}^{-1}(r) \partial_{r} f_{\ell, p}(r)-r^{-1}\right] \hat{r}_{l}+i \ell r^{-1} \hat{\phi}_{l}+i k \hat{z}_{l}\right\}\right)\right] \frac{1}{E_{r s}+\hbar c k^{\prime}}\right\} .
\end{aligned}
$$

This matrix element can be rewritten in terms of the electric-dipole and electric-dipole-electric-quadrupole polarizability tensors:

$$
\begin{aligned}
M_{f i}= & -\left[n k k^{\prime}\right]^{\frac{1}{2}}\left(\frac{\hbar c}{2 \varepsilon_{0} V}\right) f_{\ell, p}(r) e^{i k z} e^{i \ell \phi} e^{-i k^{\prime} \cdot R^{\prime}} e_{i}^{\prime} e_{j}\left(\alpha_{i j}^{t s}\left(\omega,-\omega^{\prime}\right)-A_{j i k}^{t s}\left(\omega,-\omega^{\prime}\right) i k^{\prime} \hat{k}_{k}^{\prime}\right. \\
& \left.+A_{i j k}^{t s}\left(\omega,-\omega^{\prime}\right)\left\{\left[f_{\ell, p}^{-1}(r) \partial_{r} f_{\ell, p}(r)-r^{-1}\right] \hat{r}_{k}+i \ell r^{-1} \hat{\phi}_{k}+i k \hat{z}_{k}\right\}\right),
\end{aligned}
$$

where

$$
A_{i j k}^{t s}\left(\omega,-\omega^{\prime}\right)=\sum_{r}\left[\frac{\mu_{i}^{t r} Q_{j k}^{r s}}{E_{r s}-\hbar c k}+\frac{Q_{j k}^{t r} \mu_{i}^{r s}}{E_{r s}+\hbar c k^{\prime}}\right] .
$$

The matrix element Eq. (20) is in agreement with the matrix element for circular-vortex differential scattering given by Eq. (1) in Ref. [49] (a factor of $\frac{1}{2}$ difference in the pre- multiplier stems from a different definition of the normalization constant). We have thus extracted the recently highlighted CVDS effect for chiral molecules scattering twisted 
photons. As discussed in the previous section, the optical activity polarizability tensor Eq. (21) can easily be turned into the vibrational optical activity polarizability tensor using the same methods highlighted there, namely, $A_{i j k}^{t s}\left(\omega,-\omega^{\prime}\right)=$ $\left\langle v^{\prime}\left|A_{i j k}(Q)\right| v\right\rangle$.

As a final application of our dispersion formula for twisted light we extract higher-order terms to scattering that form part of second-order corrections which have recently been studied for illumination without optical OAM [54]. In their study, Cameron and Mackinnon looked at the eight different combinations of the varying polarizability tensors, which include $A^{2}, G^{2}$, and $A G$ among more exotic interferences between $\alpha$ and higher-order polarizabilities that form the second-order corrections to zeroth-order $\alpha^{2}$ scattering. These second-order corrections are exhibited by both achiral and chiral molecules (although they do not change sign when a chiral molecule is replaced by its enantiomer), unlike the first-order corrections $\alpha A$ and $\alpha G$, which are exhibited only by chiral molecules and whose sign is dependent on molecular handedness.

Because it is now established that the scattering of twisted light through the $A$ tensor and not the $G$ tensor yields effects that are dependent on the structure of the twisted beam, and specifically the OAM, we will now extract the $A^{2}$ term from our dispersion formula (we may have equally extracted the
$A G$ to highlight our general point, though the specific form would be different). Once again, we assume the scattering of $n$ photons in the mode $(k, \eta, \ell, p)$ being scattered into a mode $\left(\boldsymbol{k}^{\prime}, \eta^{\prime}\right)$ by a molecule, and extracting the relevant terms from Eq. (14) yields the following differential cross section for $A^{2}$ scattering:

$$
\begin{aligned}
\frac{d \sigma}{d \Omega^{\prime}}\left(A^{2}\right)= & \frac{k k^{\prime 3}}{16 \pi^{2} \varepsilon_{0}^{2}} f_{\ell, p}^{2}(r) \bar{e}_{i}^{\prime} e_{j} e_{l}^{\prime} \bar{e}_{m}\left(\chi_{k} A_{i j k}^{t s}-i k^{\prime} \hat{k}_{k}^{\prime} A_{j i k}^{t s}\right) \\
& \times\left(\bar{\chi}_{n} A_{l m n}^{t s}+i k^{\prime} \hat{k}_{n}^{\prime} A_{m l n}^{t s}\right),
\end{aligned}
$$

with the frequency dependence of the polarizability tensor now implied and where we have introduced the complex vector $\chi$ as

$$
\chi=\left(\left[\frac{1}{f_{\ell, p}(r)} \partial_{r} f_{\ell, p}(r)-\frac{1}{r}\right] \hat{\boldsymbol{r}}+\frac{i \ell}{r} \hat{\boldsymbol{\phi}}+i k \hat{z}\right) .
$$

Our aim here is to highlight physics that the optical OAM of the incident beam engenders, and as such by only retaining terms that involve an $\ell$ dependence we can more clearly exhibit such differences compared to laser light that possesses no OAM $(\ell=0)$. Expanding the parentheses on the righthand side of Eq. (22) using relation (23) and retaining only the terms that have an $\ell$ dependence produces

$$
\begin{aligned}
\frac{d \sigma}{d \Omega^{\prime}}\left(\ell, A^{2}\right)= & \frac{k k^{\prime 3}}{16 \pi^{2} \varepsilon_{0}^{2}} f_{\ell, p}^{2}(r) \bar{e}_{i}^{\prime} e_{j} e^{\prime}{ }_{l} \bar{e}_{m} \frac{\ell}{r} \\
& \times\left\{A_{i j k}^{t s} A_{l m n}^{t s}\left(i\left[\frac{1}{f_{\ell, p}(r)} \partial_{r} f_{\ell, p}(r)-\frac{1}{r}\right]\left[\hat{\phi}_{k} \hat{r}_{n}-\hat{r}_{k} \hat{\phi}_{n}\right]+k\left[\hat{\phi}_{k} \hat{z}_{n}+\hat{z}_{k} \hat{\phi}_{n}\right]+\frac{\ell}{r} \hat{\phi}_{k} \hat{\phi}_{n}\right)\right. \\
& \left.-k^{\prime} \hat{\phi}_{k} \hat{k}_{n}^{\prime} A_{i j k}^{t s} A_{l n m}^{t s}-k^{\prime} \hat{\phi}_{n} \hat{k}_{k}^{\prime} A_{i k j}^{t s} A_{l m n}^{t s}\right\} .
\end{aligned}
$$

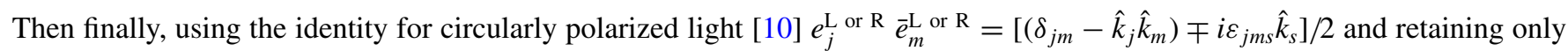
the real terms as they correspond to physically observable contributions, we find

$$
\begin{aligned}
\frac{d \sigma}{d \Omega^{\prime}}\left(\mathrm{L} \text { or R, } \ell, A^{2}\right)= & \frac{k k^{\prime 3}}{32 \pi^{2} \varepsilon_{0}^{2}} f_{\ell, p}^{2}(r) \bar{e}_{i}^{\prime} e^{\prime}{ }_{l}^{\ell} \frac{\ell}{r}\left\{\left( \pm \varepsilon_{j m s} \hat{k}_{s}\left[\frac{1}{f_{\ell, p}(r)} \partial_{r} f_{\ell, p}(r)-\frac{1}{r}\right]\left[\hat{\phi}_{k} \hat{r}_{n}-\hat{r}_{k} \hat{\phi}_{n}\right] A_{i j k}^{t s} A_{l m n}^{t s}\right)\right. \\
& \left.+\left(\delta_{j m}-\hat{k}_{j} \hat{k}_{m}\right)\left(\left[k\left[\hat{\phi}_{k} \hat{z}_{n}+\hat{z}_{k} \hat{\phi}_{n}\right]+\frac{\ell}{r} \hat{\phi}_{k} \hat{\phi}_{n}\right] A_{i j k}^{t s} A_{l m n}^{t s}-k^{\prime} \hat{\phi}_{k} \hat{k}_{n}^{\prime} A_{i j k}^{t s} A_{l n m}^{t s}-k^{\prime} \hat{\phi}_{n} \hat{k}_{k}^{\prime} A_{i k j}^{t s} A_{l m n}^{t s}\right)\right\} .
\end{aligned}
$$

Because every term in Eq. (25) has a dependence on $\ell$, it represents all of the novel contributions to $A^{2}$ scattering that are only possibly due to the incident light possessing an optical OAM - a simple Gaussian beam would not produce such interactions, for example.

Although the form of Eq. (25) is complicated, it does highlight the novelties to light-matter interactions with structured light possessing OAM, for example, can introduce. Some explicit terms of (25) are worth discussing briefly: first, the first term in brackets on the right-hand side of (25) is discriminatory with regard to the circular-polarization handedness (indicated by the \pm ), being positive for lefthanded light (L) and negative for right-handed light (R), and it is also linearly dependent on $\ell$ (and hence its sign, too). If we denote this helicity of light as $\eta= \pm 1$, we see that this first term represents a spin-orbit interac- tion of light in the sense that it is invariant under the transformation $(\eta, \ell) \rightarrow(-\eta,-\ell)$, but not $(\eta, \ell) \rightarrow(-\eta, \ell)$ or $(\eta, \ell) \rightarrow(\eta,-\ell)$.

Another important point in the analysis for structured beams is that all of the terms stemming from E2 interactions display dependencies on the unit vectors of the input light, and integration of the total beam profile over those with a linear dependence on the unit vectors $\hat{\phi}_{k}, \hat{r}_{n}$ will produce a null result due to the cylindrical symmetry of the transverse profile. This therefore means that any effect stemming from these terms will require the ability to resolve individual sections of the beam profile [55], or similarly, carrying out experiments with the beam positioned off-axis with respect to the sample being probed.

A term in Eq. (25) which will not require this more intricate experimental study is the $\ell^{2} r^{-2} \hat{\phi}_{k} \hat{\phi}_{n}$ term, which due to its 
quadratic dependence on $\ell$ clearly has the capacity to contribute significantly to the scattering of light for high values of $\ell$ (which theoretically is an unbounded integer, and the newly discovered spiral phase mirror has allowed experimentally realized values to reach 5050 for a vortex beam [56]) - no such enhanced scattering mechanism exists for beams without OAM. Furthermore, it also possesses an $r^{-2}$ dependence which is acutely linked to the transverse intensity distribution of the light beam, and so any relative enhancement compared to unstructured light will strongly depend on where the scatterer is positioned in the beam profile.

In standard Laguerre-Gaussian vortices the intensity distribution varies for different values of $\ell$. However, this potentially complicating factor in experiments can be overcome by using the so-called "perfect" optical vortices [57,58], which have constant intensity distributions that are independent of $\ell$. Indeed, these perfect vortices will play a prominent role in any future spectroscopic applications of twisted light beams in the chemical and physical sciences, especially as they are now being produced with large topological charges [59].

We now extract from Eq. (15) an example of an interference between two pure-multipole moment polarizability tensors contributing to second-order scattering, namely, the dominant electric-dipole molecular polarizability Eq. (17) coupling with the pure electric-quadrupole polarizability tensor $\Theta_{k l m n}^{t s}\left(\omega,-\omega^{\prime}\right)$ :

$$
\begin{aligned}
\frac{d \sigma}{d \Omega^{\prime}}(\ell, \alpha \Theta)= & \frac{k k^{\prime 4}}{32 \pi^{2} \varepsilon_{0}^{2}} f_{\ell, p}^{2}(r) \bar{e}_{i}^{\prime} e_{k}^{\prime}\left(\delta_{j m}-\hat{k}_{j} \hat{k}_{m}\right) \\
& \times \frac{\ell}{r} \hat{k}_{l}^{\prime} \hat{\phi}_{n}\left[\alpha_{i j}^{t s} \Theta_{k l m n}^{t s}+\alpha_{k m}^{t s} \Theta_{i l j n}^{t s}\right],
\end{aligned}
$$

where the pure electric quadrupole polarizability tensor is defined as

$$
\Theta_{k l m n}^{t s}\left(\omega,-\omega^{\prime}\right)=\sum_{r}\left[\frac{Q_{k l}^{t r} Q_{m n}^{r s}}{E_{r s}-\hbar c k}+\frac{Q_{m n}^{t r} Q_{k l}^{r s}}{E_{r s}+\hbar c k^{\prime}}\right] .
$$

In extracting Eq. (26) from our Kramers-Heisenberg dispersion formula for twisted light Eq. (14) we have once again retained terms with an OAM dependence only through $\ell$ for reasons previously stated.

Finally, the results of Eqs. (25) and (26) are currently applicable to molecular systems that are oriented, or in the so-called locked-in state for the bulk phase, e.g., a solid. In order to produce a result applicable to scattering by molecules in the liquid or gas phases it is necessary to take random molecular tumbling into account. This involves the rotational averaging of the molecular-polarizability tensors using standard techniques [60]. In both cases this involves employing the result for the average of a sixth-rank Cartesian tensor. For $A^{2}$ scattering this average is of the form $\left\langle A_{i j k} A_{l m n}\right\rangle$, while for $\alpha \Theta$ scattering we require the average $\left\langle\alpha_{i j} \Theta_{k l m n}\right\rangle$. Details are provided in Appendix B. It is important to state here that first the results do not vanish upon averaging (since it comprises isotropic tensors of 6th rank, which have no antisymmetric part) and that the general physical characteristics of $A^{2}$ scattering, such as the discriminatory and $\ell^{2}$ dependent properties we have drawn out for the oriented case, are still observable for the fully averaged form of Eq. (25) for certain scattering angle and scattered light polarization analyses.

\section{DISCUSSION AND CONCLUSION}

The Kramers-Heisenberg dispersion formula and the subsequent evaluation of a fully quantum form derived by Dirac were extremely important steps forward in the theory of light-matter interactions and provided insight into foundational principles between the classical and quantum theories of radiation. In this article we have taken this well-known formula in optics and rederived it in a form that can account for the scattering of twisted light possessing optical OAM, producing a Kramers-Heisenberg dispersion formula that can yield scattering cross sections and results of importance to one of the most research-intensive areas of modern optics. It is to be emphasized that the formulas derived are of a general form, applicable to any type of molecular matter that supports the transition multipoles (electric dipole, magnetic dipole, etc.) relevant to any specific multipolar contribution to the scattering. Moreover, we have explicitly accounted for higher-order multipolar contributions beyond the dominant electric-dipole interactions with the field in order to account for chiral light-matter interactions, a topic of extreme interest in the last few years due to the possibilities afforded by optically active molecules engaging with optical OAM. It has been demonstrated how the relevant multipolar contributions can easily be extracted from our dispersion formula in order to account for the twisted light forms of Rayleigh and Raman scattering, Rayleigh and Raman optical activity, and the newly discovered circular-vortex differential scattering forms of Rayleigh and Raman optical activity and secondorder scattering contributions such as the explicitly derived $A^{2}$ and $\alpha \Theta$ scattering terms. While expected, it has been explicitly shown that the major aspects of optical dispersion are not changed much by the involvement of structured light. Importantly, however, the scattering cross sections are greatly altered if E2 moments are engaged due to their unique sensitivity to the phase properties of the input beam, revealing numerous novel interactions with the light's optical OAM, including the possibility of significantly enhanced scattering. We reemphasize that both the helical-phase and intensity distribution offer no novel effects for solely dipole (electric and magnetic) contributions to scattering. The electric quadrupole transition moment contributions, however, must be included for novel effects to arise. In these quadrupole interactions, the potential for enhanced scattering arises solely from the $\ell$-dependent terms unique to structured light. In general, the $\ell$-dependent terms scale versus standard unstructured light scattering as $\approx(k r)^{-1}$, so for positions in the beam where we simply fix $k r=1$, the simple and indicative observation can be made that any $\ell$-dependent term scattering enhancements will increase linearly as the OAM increases, relative to the unstructured light contributions to scattering at $k r=1$. (For $\ell=1$ the increased scattering of twisted light compared to unstructured light is practically equal for a scatterer at $k r=1$, the overall scattering being their sum).

Our Kramers-Heisenberg dispersion formula was derived for the scattering of an incident Laguerre-Gaussian beam of 
twisted light, which is a solution to the scalar wave equation under the paraxial approximation (i.e., the profile of the beam varies slowly in the $z$ direction). The distinction between paraxial and nonparaxial optics is extremely important in considering both the properties of the laser beam itself upon propagation [61] and also the light-matter interactions. Nonparaxial light is particularly important, as it is the main source of spin-orbit interactions of light (SOI), responsible for many novel optical interactions on the subwavelength scale of nanooptics and photonics [62]. Indeed, scattering itself is a method of producing SOI, along with strongly focused beams of light. Future studies will aim to look at the role nonparaxial light and SOI can have in molecular studies of the dispersion formula.

\section{ACKNOWLEDGMENTS}

We would like to thank David L. Andrews for stimulating discussions and David S. Bradshaw for comments. K.A.F. would like to thank the Leverhulme Trust for funding him through a Leverhulme Early Career Fellowship.

\section{APPENDIX A: MULTIPOLAR MATRIX ELEMENTS FOR ABSORPTION AND EMISSION OF TWISTED PHOTONS}

In Sec. III we derived the matrix element for the scattering of twisted light Eq. (13) using second-order perturbation theory Eq. (6). The derivation involves the product of two singlephoton matrix elements [i.e., the numerator of Eq. (6)]. These single-photon matrix elements involve the absorption and emission of twisted photons and are first-order optical pro-

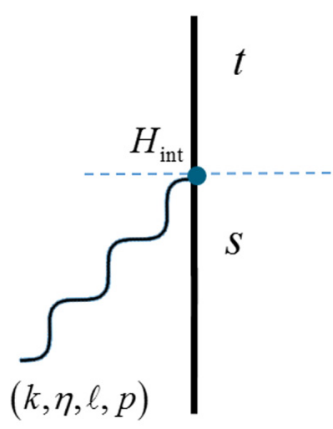

Absorption

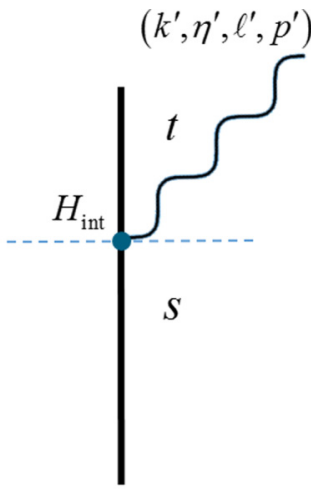

Emission
FIG. 3. Feynman graphs to aid the calculation of single-photon absorption of a photon in mode $(k, \eta, \ell, p)$ and emission of a photon in mode $\left(k^{\prime}, \eta^{\prime}, \ell^{\prime}, p^{\prime}\right)$ in diagrammatic time-dependent perturbation theory. Time progresses in the upward direction, with space along the horizontal axis.

cesses (Fig. 3). By using Eq. (1) and first-order perturbation theory $M_{f i}=\left\langle f\left|H_{\text {int }}\right| i\right\rangle$ we can compute the required singlephoton matrix elements used in the derivation of Eq. (13).

In both cases the molecule is taken to be in an initial state $|s\rangle$ while the initial radiation field state is $|n(k, \eta, \ell, p)\rangle$ for absorption and $\left|n^{\prime}\left(k^{\prime}, \eta^{\prime}, \ell^{\prime}, p^{\prime}\right)\right\rangle$ for emission (the primes on the latter indicating emission). The first-order matrix elements for both processes may be partitioned into their distinct multipolar contributions due to the form of Eq. (1). For single-photon absorption producing a final system state $|t ;(n-1)(k, \eta, \ell, p)\rangle$, we have for the matrix elements

$$
\begin{aligned}
& \left\langle t ;(n-1)(k, \eta, \ell, p)\left|-\varepsilon_{0}^{-1} \boldsymbol{\mu}(\xi) \cdot \boldsymbol{d}^{\perp}\left(\boldsymbol{R}_{\xi}\right)\right| s ; n(k, \eta, \ell, p)\right\rangle=-i\left(\frac{n \hbar c k}{2 \varepsilon_{0} V}\right)^{\frac{1}{2}} f_{\ell, p}(r) e_{i} \mu_{i}^{t s} e^{i k z} e^{i \ell \phi}, \\
& \left\langle t ;(n-1)(k, \eta, \ell, p)\left|-\boldsymbol{m}(\xi) \cdot \boldsymbol{b}\left(\boldsymbol{R}_{\xi}\right)\right| s ; n(k, \eta, \ell, p)\right\rangle=-i\left(\frac{n \hbar k}{2 \varepsilon_{0} c V}\right)^{\frac{1}{2}} f_{\ell, p}(r) b_{i} m_{i}^{t s} e^{i k z} e^{i \ell \phi},
\end{aligned}
$$

and

$$
\begin{aligned}
& \left\langle t ;(n-1)(k, \eta, \ell, p)\left|-\varepsilon_{0}^{-1} Q_{i j}(\xi) \nabla_{j} d_{i}^{\perp}\left(\boldsymbol{R}_{\xi}\right)\right| s ; n(k, \eta, \ell, p)\right\rangle \\
& \quad=-i\left(\frac{n \hbar c k}{2 \varepsilon_{0} V}\right)^{\frac{1}{2}} f_{\ell, p}(r) e_{i} Q_{i j}^{t s}\left\{\left[f_{\ell, p}^{-1}(r) \partial_{r} f_{\ell, p}(r)-r^{-1}\right] \hat{r}_{j}+i \ell r^{-1} \hat{\phi}_{j}+i k \hat{z}_{j}\right\} e^{i k z} e^{i \ell \phi},
\end{aligned}
$$

where we have dropped the mode dependencies on the polarization vectors for notational brevity. Expression (A3) has been secured by using the key result of the gradient operator acting upon the structured field [53], unique to E2 interactions at this order of multipolar expansion:

$$
\nabla_{j} d_{i}^{\perp} \approx \nabla_{j} e_{i} f_{\ell, p}(r) e^{(i k z+i \ell \phi)}=e_{i}\left[\hat{r}_{j} \partial_{r} f_{\ell, p}(r) e^{(i k z+i \ell \phi)}+f_{\ell, p}(r) \frac{1}{r}\left(i \ell \hat{\phi}_{j}-\hat{r}_{j}\right) e^{(i k z+i \ell \phi)}+f_{\ell, p}(r) i k \hat{z}_{j} e^{(i k z+i \ell \phi)}\right] .
$$

In the limit where $\ell=0$, Eq. (A4) reduces to a $z$-propagating Gaussian beam with a typical Gaussian distribution function $f_{0,0}(r)$ given by the appropriately modified Eq. (4), and most importantly, the middle term in brackets in (A4), which represents the helical-phase gradient only present in OAM beams vanishes. 
The multipolar contributions to the matrix elements for the emission of light from the initial state given previously to the final state $\left|t ;\left(n^{\prime}+1\right)\left(k^{\prime}, \eta^{\prime}, \ell^{\prime}, p^{\prime}\right)\right\rangle$ may similarly be derived, yielding

$$
\begin{gathered}
\left\langle t ;\left(n^{\prime}+1\right)\left(k^{\prime}, \eta^{\prime}, \ell^{\prime}, p^{\prime}\right)\left|-\varepsilon_{0}^{-1} \boldsymbol{\mu}(\xi) \cdot \boldsymbol{d}^{\perp}\left(\boldsymbol{R}_{\xi}\right)\right| s ; n^{\prime}\left(k^{\prime}, \eta^{\prime}, \ell^{\prime}, p^{\prime}\right)\right\rangle=i\left(\frac{\left(n^{\prime}+1\right) \hbar c k^{\prime}}{2 \varepsilon_{0} V}\right)^{\frac{1}{2}} \bar{f}_{\ell^{\prime}, p^{\prime}}(r){\overline{e^{\prime}}}_{i} \mu_{i}^{t s} e^{-i k^{\prime} z} e^{-i \ell^{\prime} \phi}, \\
\left\langle t ;\left(n^{\prime}+1\right)\left(k^{\prime}, \eta^{\prime}, \ell^{\prime}, p^{\prime}\right)\left|-\boldsymbol{m}(\xi) \cdot \boldsymbol{b}\left(\boldsymbol{R}_{\xi}\right)\right| s ; n^{\prime}\left(k^{\prime}, \eta^{\prime}, \ell^{\prime}, p^{\prime}\right)\right\rangle=i\left(\frac{\left(n^{\prime}+1\right) \hbar k^{\prime}}{2 \varepsilon_{0} c V}\right)^{\frac{1}{2}} \bar{f}_{\ell^{\prime}, p^{\prime}}(r) \bar{b}_{i}^{\prime} m_{i}^{t s} e^{-i k^{\prime} z} e^{-i \ell^{\prime} \phi},
\end{gathered}
$$

and

$$
\begin{aligned}
& \left\langle t ;\left(n^{\prime}+1\right)\left(k^{\prime}, \eta^{\prime}, \ell^{\prime}, p_{1}^{\prime}\right)\left|-\varepsilon_{0}^{-1} Q_{i j}(\xi) \nabla_{j} d_{i}^{\perp}\left(\boldsymbol{R}_{\xi}\right)\right| s ; n^{\prime}\left(k^{\prime}, \eta^{\prime}, \ell^{\prime}, p^{\prime}\right)\right\rangle \\
& \quad=i\left(\frac{\left(n^{\prime}+1\right) \hbar c k^{\prime}}{2 \varepsilon_{0} V}\right)^{\frac{1}{2}} f_{\ell^{\prime}, p^{\prime}}(r) \bar{e}_{i}^{\prime} Q_{i j}^{t s}\left\{\left[f_{\ell^{\prime}, p^{\prime}}^{-1}(r) \partial_{r} f_{\ell^{\prime}, p^{\prime}}(r)-r^{-1}\right] \hat{r}_{j}-i \ell^{\prime} r^{-1} \hat{\phi}_{j}-i k^{\prime} \hat{z}_{j}\right\} e^{-i k^{\prime} z} e^{-i \ell^{\prime} \phi} .
\end{aligned}
$$

\section{APPENDIX B: ROTATIONAL AVERAGING OF $A^{2}$ AND $\alpha \Theta$ CONTRIBUTIONS TO SCATTERING}

In Sec. $\mathrm{V}$ of the main article we derive the $A^{2}$ and $\alpha \Theta$ contributions to the scattering of twisted light. The results, Eqs. (25) and (26), respectively, as it stands are currently applicable to anisotropic systems, such as oriented molecules, and therefore to make the result applicable to isotropic systems, such as liquids and gases, we require a full rotational average. This is achieved through standard techniques [60] and in this case involves the 6th-rank averaging $I^{(6)}$ of the molecular parts $\left\langle A_{i j k} A_{l m n}\right\rangle$ and $\left\langle\alpha_{i j} \Theta_{k l m n}\right\rangle$.

We first calculate the average of the $A^{2}$ contribution to scattering. Important in the ensuing average is that $A_{i j k}$ is $j, k$ symmetric due to the $j, k$-symmetric property of the electric quadrupole moment $Q_{j k}$, and furthermore, $Q_{j k}$ is also traceless, namely, $Q_{j j}=0$. In the average Latin indices refer to space-fixed frames while Greek indices correspond to molecule-fixed frames. We thus require the following averages: $\left\langle A_{i j k} A_{l m n}\right\rangle=I^{(6)} A_{\lambda \mu \nu} A_{\rho \sigma \tau}$. The full form of $I^{(6)}$ is a $15 \times 15$ matrix of coefficients together with a space-fixed frame row and body-fixed frame column vectors given by Eq. (B1). It is constructed from various combinations of the isotropic second-rank Kronecker $\delta$ tensors $\delta_{i j}$ and $\delta_{\lambda \mu}$, which form so-called isomers:

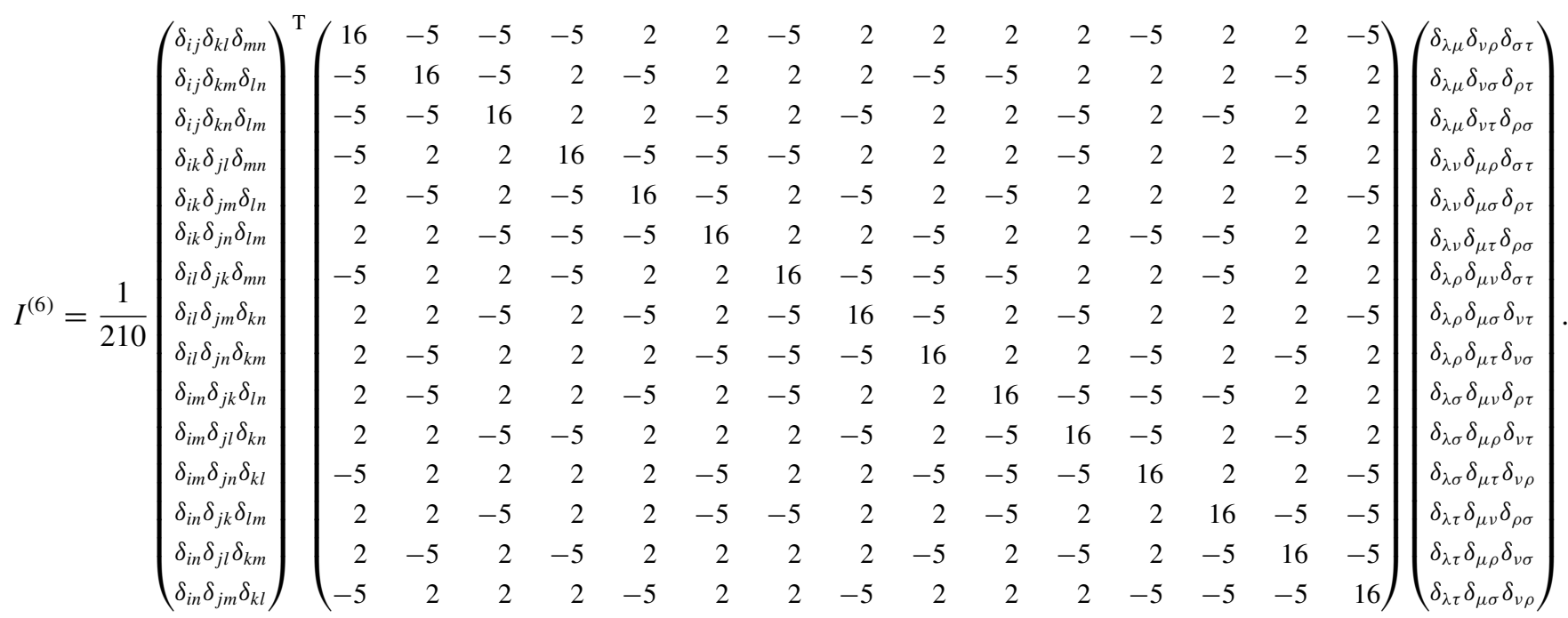

Because of the symmetry properties of the $A_{i j k}$ tensor mentioned above, numerous isomers present in the full form of $I^{(6)}$ vanish. Namely, $A_{\lambda \mu \nu} A_{\rho \sigma \tau}$ vanishes when $\mu=\nu$ and $\sigma=\tau$. Consequently, the 15 isomers in (B1) are reduced to 10 (i.e., we produce a $10 \times 10$ matrix), where the $1 \mathrm{st}, 4 \mathrm{th}, 7 \mathrm{th}, 10 \mathrm{th}$, and 13 th rows in (B1) need not be evaluated. Carrying out the many calculations and tensor contractions of the reduced $10 \times 10$ matrix $I^{\prime(6)}$ gives

$$
\begin{aligned}
\left\langle A_{i j k} A_{l m n}\right\rangle= & I^{\prime(6)} A_{\lambda \mu \nu} A_{\rho \sigma \tau}=\frac{1}{210}\left\{\left(\delta_{i j} \delta_{k m} \delta_{l n}+\delta_{i j} \delta_{k n} \delta_{l m}\right)\left(8 A_{\lambda \lambda \mu} A_{\nu v \mu}-3\left[A_{\lambda \mu \nu} A_{\lambda \mu \nu}+A_{\lambda \mu \nu} A_{\mu \nu \lambda}\right]+4 A_{\lambda \mu \nu} A_{\nu \lambda \mu}\right)\right. \\
& +\left(\delta_{i k} \delta_{j m} \delta_{l n}+\delta_{i k} \delta_{j n} \delta_{l m}\right)\left(8 A_{\lambda \lambda \mu} A_{\nu v \mu}-3\left[A_{\lambda \mu \nu} A_{\lambda \mu \nu}+A_{\lambda \mu \nu} A_{\nu \lambda \mu}\right]+4 A_{\lambda \mu \nu} A_{\mu \nu \lambda}\right) \\
& +\left(\delta_{i l} \delta_{j m} \delta_{k n}+\delta_{i l} \delta_{j n} \delta_{k m}\right)\left(-6 A_{\lambda \lambda \mu} A_{\nu \nu \mu}+11 A_{\lambda \mu \nu} A_{\lambda \mu \nu}-3\left[A_{\lambda \mu \nu} A_{\mu \nu \lambda}+A_{\lambda \mu \nu} A_{\nu \lambda \mu}\right]\right) \\
& +\left(\delta_{i m} \delta_{j l} \delta_{k n}+\delta_{i m} \delta_{j n} \delta_{k l}+\delta_{i n} \delta_{j l} \delta_{l k}\right)\left(A_{\lambda \lambda \mu} A_{\nu v \mu}-3\left[A_{\lambda \mu \nu} A_{\lambda \mu \nu}+A_{\lambda \mu \nu} A_{\nu \lambda \mu}\right]+11 A_{\lambda \mu \nu} A_{\mu \nu \lambda}\right) \\
& \left.+\delta_{i n} \delta_{j m} \delta_{k l}\left(A_{\lambda \lambda \mu} A_{\nu \nu \mu}-3\left[A_{\lambda \mu \nu} A_{\lambda \mu \nu}+A_{\lambda \mu \nu} A_{\mu \nu \lambda}\right]+11 A_{\lambda \mu \nu} A_{\nu \lambda \mu}\right)\right\}
\end{aligned}
$$


The molecular average (B2) can be used on the first and second terms in Eq. (25) that are dependent on $\left\langle A_{i j k} A_{l m n}\right\rangle$. However, we also require the averages $\left\langle A_{i j k} A_{l n m}\right\rangle$ and $\left\langle A_{i k j} A_{l m n}\right\rangle$ in order to calculate the averaged result of the final two terms in Eq. (25). By interchanging $m \leftrightarrow n$ in (B2) we obtain the required tensor average through the transformation $\left\langle A_{i j k} A_{l m n}\right\rangle \rightarrow\left\langle A_{i j k} A_{l n m}\right\rangle$ and similarly, $k \leftrightarrow j$ yields $\left\langle A_{i j k} A_{l m n}\right\rangle \rightarrow\left\langle A_{i k j} A_{l m n}\right\rangle$. The results are then fed back into Eq. (25), whereby through significant calculational effort the space-fixed frame tensors from the orientational averages [the Kronecker $\delta$ terms in (B2)] are contracted with the geometric tensors, e.g., $\bar{e}_{i}^{\prime} e_{l}^{\prime} \varepsilon_{j m s} \hat{k}_{s}\left[\hat{\phi}_{k} \hat{r}_{n}-\hat{r}_{k} \hat{\phi}_{n}\right]$ for the first term in Eq. (25), to yield the fully rotationally averaged $A^{2}$ scattering cross section as

$$
\begin{aligned}
& \left\langle\frac{d \sigma}{d \Omega^{\prime}}\right\rangle\left(\mathrm{L} \text { or } \mathrm{R}, \ell, A^{2}\right)=\frac{k k^{\prime 3} f_{\ell, p}^{2}(r)}{6720 \pi^{2} \varepsilon_{0}^{2}} \frac{\ell}{r}\left\{\left( \pm\left[\left(\left[\overline{\boldsymbol{e}}^{\prime} \cdot(\hat{\boldsymbol{\phi}} \times \hat{z})\right]\left(\boldsymbol{e}^{\prime} \cdot \hat{\boldsymbol{r}}\right)-\left[\overline{\boldsymbol{e}}^{\prime} \cdot(\hat{\boldsymbol{r}} \times \hat{z})\right]\left(\boldsymbol{e}^{\prime} \cdot \hat{\boldsymbol{\phi}}\right)\right) a\right.\right.\right. \\
& \left.\left.+\left(\left[\boldsymbol{e}^{\prime} \cdot(\hat{\boldsymbol{\phi}} \times \hat{\boldsymbol{z}})\right]\left(\overline{\boldsymbol{e}^{\prime}} \cdot \hat{\boldsymbol{r}}\right)-\left[\boldsymbol{e}^{\prime} \cdot(\hat{\boldsymbol{r}} \times \hat{\boldsymbol{z}})\right]\left(\overline{\boldsymbol{e}^{\prime}} \cdot \hat{\boldsymbol{\phi}}\right)\right) b+2[\hat{\boldsymbol{r}} \cdot(\hat{\boldsymbol{\phi}} \times \hat{\boldsymbol{z}})] c\right]\left[\frac{1}{f_{\ell, p}(r)} \partial_{r} f_{\ell, p}(r)-\frac{1}{r}\right]\right) \\
& +\left[\left(\overline{\boldsymbol{e}}^{\prime} \cdot \hat{\boldsymbol{\phi}}\right)\left(\boldsymbol{e}^{\prime} \cdot \hat{z}\right) a+2\left[\left(\overline{\boldsymbol{e}}^{\prime} \cdot \hat{\boldsymbol{\phi}}\right)\left(\boldsymbol{e}^{\prime} \cdot \hat{z}\right)+\left(\overline{\boldsymbol{e}^{\prime}} \cdot \hat{z}\right)\left(\boldsymbol{e}^{\prime} \cdot \hat{\boldsymbol{\phi}}\right)\right] d\right. \\
& \left.+\left(\overline{\boldsymbol{e}^{\prime}} \cdot \hat{z}\right)\left(\boldsymbol{e}^{\prime} \cdot \hat{\boldsymbol{\phi}}\right) e+\left(\overline{\boldsymbol{e}^{\prime}} \cdot \hat{z}\right)\left(\boldsymbol{e}^{\prime} \cdot \hat{\boldsymbol{\phi}}\right) f\right] k-\left[\left(1-\left|\boldsymbol{e}^{\prime} \cdot \hat{z}\right|^{2}\right)\left(\hat{\boldsymbol{k}}^{\prime} \cdot \hat{\boldsymbol{\phi}}\right) a-\left(\overline{\boldsymbol{e}^{\prime}} \cdot \hat{\boldsymbol{\phi}}\right)\left(\boldsymbol{e}^{\prime} \cdot \hat{z}\right)\left(\hat{\boldsymbol{k}}^{\prime} \cdot \hat{z}\right) f\right. \\
& \left.-\left(\boldsymbol{e}^{\prime} \cdot \hat{\boldsymbol{\phi}}\right)\left(\overline{\boldsymbol{e}^{\prime}} \cdot \hat{z}\right)\left(\hat{\boldsymbol{k}}^{\prime} \cdot \hat{z}\right) g+3\left(\hat{\boldsymbol{k}}^{\prime} \cdot \hat{\boldsymbol{\phi}}\right) c\right]-\left[\left(1-\left|\boldsymbol{e}^{\prime} \cdot \hat{z}\right|^{2}\right)\left(\hat{\boldsymbol{k}}^{\prime} \cdot \hat{\boldsymbol{\phi}}\right) b-\left(\boldsymbol{e}^{\prime} \cdot \hat{\boldsymbol{\phi}}\right)\left(\overline{\boldsymbol{e}^{\prime}} \cdot \hat{z}\right)\left(\hat{\boldsymbol{k}}^{\prime} \cdot \hat{z}\right) f\right. \\
& \left.\left.-\left(\overline{\boldsymbol{e}^{\prime}} \cdot \hat{\boldsymbol{\phi}}\right)\left(\boldsymbol{e}^{\prime} \cdot \hat{z}\right)\left(\hat{\boldsymbol{k}}^{\prime} \cdot \hat{z}\right) g+3\left(\hat{\boldsymbol{k}}^{\prime} \cdot \hat{\boldsymbol{\phi}}\right) c\right] k^{\prime}\right\}
\end{aligned}
$$

where

$$
\begin{gathered}
a=\left(9 A_{\lambda \lambda \mu} A_{\nu v \mu}-6 A_{\lambda \mu \nu} A_{\lambda \mu \nu}+8 A_{\lambda \mu \nu} A_{\mu \nu \lambda}+A_{\lambda \mu \nu} A_{\nu \lambda \mu}\right), \\
b=\left(9 A_{\lambda \lambda \mu} A_{\nu v \mu}-6 A_{\lambda \mu \nu} A_{\lambda \mu \nu}+15 A_{\lambda \mu \nu} A_{\mu \nu \lambda}-6 A_{\lambda \mu \nu} A_{\nu \lambda \mu}\right), \\
c=\left(-6 A_{\lambda \lambda \mu} A_{\nu v \mu}+11 A_{\lambda \mu \nu} A_{\lambda \mu \nu}-3\left[A_{\lambda \mu \nu} A_{\mu \nu \lambda}+A_{\lambda \mu \nu} A_{\nu \lambda \mu}\right]\right), \\
d=\left(9 A_{\lambda \lambda \mu} A_{\nu v \mu}-6 A_{\lambda \mu \nu} A_{\lambda \mu \nu}+8 A_{\lambda \mu \nu} A_{\nu \lambda \mu}+A_{\lambda \mu \nu} A_{\mu \nu \lambda}\right), \\
e=\left(A_{\lambda \lambda \mu} A_{\nu v \mu}-3\left[A_{\lambda \mu \nu} A_{\lambda \mu \nu}+A_{\lambda \mu \nu} A_{\nu \lambda \mu}\right]+11 A_{\lambda \mu \nu} A_{\mu \nu \lambda}\right), \\
f=\left(8 A_{\lambda \lambda \mu} A_{\nu \nu \mu}-3\left[A_{\lambda \mu \nu} A_{\lambda \mu \nu}+A_{\lambda \mu \nu} A_{\nu \lambda \mu}\right]+4 A_{\lambda \mu \nu} A_{\mu \nu \lambda}\right),
\end{gathered}
$$

and

$$
g=\left(A_{\lambda \lambda \mu} A_{\nu v \mu}-3\left[A_{\lambda \mu \nu} A_{\lambda \mu \nu}+A_{\lambda \mu \nu} A_{\mu \nu \lambda}\right]+11 A_{\lambda \mu \nu} A_{\nu \lambda \mu}\right) .
$$

In deriving the form of (B3) we have taken into account the paraxial nature of the twisted light, namely, that $\hat{\boldsymbol{k}}=\hat{z}$, and also that $\hat{z} \cdot \hat{\boldsymbol{\phi}}=0$ and $\hat{z} \cdot \hat{\boldsymbol{r}}=0$.

The calculations in the average required for $\left\langle\alpha_{i j} \Theta_{k l m n}\right\rangle=I^{(6)} \alpha_{\lambda \mu} \Theta_{\nu \rho \sigma \tau}$ follow similar lines, as it too is a 6th-rank tensor average. The full form of $I^{(6)}$ is reduced again to a $10 \times 10$ matrix due to the symmetry properties of $\Theta_{k l m n}$; however, it takes on a different form $I^{\prime \prime(6)}$ as in this case the 1st, 4th, 7th, 12th, and 15th rows in Eq. (B1) vanish when $v=\rho$ and $\sigma=\tau$. The equivalent form of (B2) for $\left\langle\alpha_{i j} \Theta_{k l m n}\right\rangle$ is seen to be

$$
\begin{aligned}
\left\langle\alpha_{i j} \Theta_{k l m n}\right\rangle= & I^{\prime \prime}(6) \alpha_{\lambda \mu} \Theta_{\nu \rho \sigma \tau}=\frac{1}{210}\left\{\left(\delta_{i j} \delta_{k m} \delta_{l n}+\delta_{i j} \delta_{k n} \delta_{l m}\right)\left(11 \alpha_{\lambda \lambda} \Theta_{\mu \nu \mu \nu}-6\left[\alpha_{\lambda \mu} \Theta_{\lambda \nu \mu \nu}+\alpha_{\lambda \mu} \Theta_{\mu \nu \lambda \nu}\right]\right)\right. \\
& +\left(\delta_{i k} \delta_{j m} \delta_{l n}+\delta_{i k} \delta_{j n} \delta_{l m}+\delta_{i l} \delta_{j m} \delta_{k n}+\delta_{i l} \delta_{j n} \delta_{k m}\right)\left(-3 \alpha_{\lambda \lambda} \Theta_{\mu \nu \mu \nu}+8 \alpha_{\lambda \mu} \Theta_{\lambda \nu \mu \nu}+\alpha_{\lambda \mu} \Theta_{\mu \nu \lambda \nu}\right) \\
& \left.+\left(\delta_{i m} \delta_{j k} \delta_{l n}+\delta_{i m} \delta_{j l} \delta_{k n}+\delta_{i n} \delta_{j k} \delta_{l m}+\delta_{i n} \delta_{j l} \delta_{k m}\right)\left(-3 \alpha_{\lambda \lambda} \Theta_{\mu \nu \mu \nu}+\alpha_{\lambda \mu} \Theta_{\lambda \nu \mu \nu}+8 \alpha_{\lambda \mu} \Theta_{\mu \nu \lambda \nu}\right)\right\} .
\end{aligned}
$$

We may find $\left\langle\alpha_{k m} \Theta_{i l j n}\right\rangle$ by making the following replacements in (B11): $i \leftrightarrow k$ and $m \leftrightarrow j$. Inserting both $\left\langle\alpha_{i j} \Theta_{k l m n}\right\rangle$ and $\left\langle\alpha_{k m} \Theta_{i l j n}\right\rangle$ into Eq. (26) and contracting the space-fixed tensors with the geometric tensors yields the fully averaged $\alpha \Theta$ contribution to scattering as

$$
\left\langle\frac{d \sigma}{d \Omega^{\prime}}\right\rangle(\ell, \alpha \Theta)=\frac{k k^{\prime 3}}{3360 \pi^{2} \varepsilon_{0}^{2}} f_{\ell, p}^{2}(r) \frac{\ell}{r}\left\{\left[1-\left|\boldsymbol{e}^{\prime} \cdot \hat{z}\right|^{2}\left(\boldsymbol{k}^{\prime} \cdot \hat{\boldsymbol{\phi}}\right)-\left(\overline{\boldsymbol{e}^{\prime}} \cdot \hat{z}\right)\left(\boldsymbol{e}^{\prime} \cdot \hat{\boldsymbol{\phi}}\right)\left(\hat{z} \cdot \boldsymbol{k}^{\prime}\right)\right] a^{\prime}+3\left(\boldsymbol{k}^{\prime} \cdot \hat{\boldsymbol{\phi}}\right) b^{\prime}-2\left(\overline{\boldsymbol{e}^{\prime}} \cdot \hat{\boldsymbol{\phi}}\right)\left(\boldsymbol{e}^{\prime} \cdot \hat{\boldsymbol{k}}\right)\left(\hat{z} \cdot \boldsymbol{k}^{\prime}\right) c^{\prime}\right\},
$$

where

$$
\begin{aligned}
& a^{\prime}=\left(8 \alpha_{\lambda \lambda} \Theta_{\mu \nu \mu \nu}-5 \alpha_{\lambda \mu} \Theta_{\lambda \nu \mu \nu}+2 \alpha_{\lambda \mu} \Theta_{\mu \nu \lambda \nu}\right), \\
& b^{\prime}=\left(-3 \alpha_{\lambda \lambda} \Theta_{\mu \nu \mu \nu}+8 \alpha_{\lambda \mu} \Theta_{\lambda \nu \mu \nu}+\alpha_{\lambda \mu} \Theta_{\mu \nu \lambda \nu}\right), \\
& c^{\prime}=\left(-3 \alpha_{\lambda \lambda} \Theta_{\mu \nu \mu \nu}+\alpha_{\lambda \mu} \Theta_{\lambda \nu \mu \nu}+8 \alpha_{\lambda \mu} \Theta_{\mu \nu \lambda \nu}\right) .
\end{aligned}
$$


The fully rotationally averaged scattering cross sections Eqs. (B3) and (B12) can then be taken further by studying scattering at specific angles and resolving the output polarization. A common example is studying right-angled scattering $\boldsymbol{k} \cdot \boldsymbol{k}^{\prime}=0$ and resolving the scattered light polarization in either the $\boldsymbol{k} \boldsymbol{k}^{\prime}$ plane $\left(\boldsymbol{e}^{\prime}(\|)\right)$ or normal to it $\boldsymbol{e}^{\prime}(\perp)$.

[1] P. Nelson, From Photon to Neuron: Light, Imaging, Vision (Princeton University Press, Princeton, NJ, 2017).

[2] L. D. Barron, L. Hecht, E. W. Blanch, and A. F. Bell, Prog. Biophys. Mol. Biol. 73, 1 (2000).

[3] L. D. Barron, Biomed. Spectrosc. Imaging 4, 223 (2015).

[4] P. M. Carvalho, M. R. Felício, N. C. Santos, S. Gonçalves, and M. M. Domingues, Front. Chem. 6, 237 (2018).

[5] N. N. Boustany, S. A. Boppart, and V. Backman, Annu. Rev. Biomed. Eng. 12, 285 (2010).

[6] Z. A. Steelman, D. S. Ho, K. K. Chu, and A. Wax, Optica 6, 479 (2019).

[7] D. L. Andrews and D. S. Bradshaw, Optical Nanomanipulation (Morgan \& Claypool Publishers, San Rafael, CA, 2017).

[8] C. Bradac, Adv. Opt. Mater. 6, 1800005 (2018).

[9] D. L. Andrews and P. Allcock, Optical Harmonics in Molecular Systems: Quantum Electrodynamical Theory (Wiley-VCH, Weinheim, 2002).

[10] D. P. Craig and T. Thirunamachandran, Molecular Quantum Electrodynamics: An Introduction to Radiation-Molecule Interactions (Courier Corporation, New York, 1998).

[11] A. Salam, Molecular Quantum Electrodynamics: Long-Range Intermolecular Interactions (John Wiley \& Sons, Hoboken, NJ, 2010).

[12] H. A. Kramers and W. Heisenberg, Z. Für Phys. 31, 681 (1925).

[13] G. Breit, Rev. Mod. Phys. 4, 504 (1932).

[14] P. A. M. Dirac, Proc. R. Soc. London, Ser. A 114, 710 (1927).

[15] W. P. Healy, Phys. Rev. A 16, 1568 (1977).

[16] W. P. Healy and R. G. Woolley, J. Phys. B: At. Mol. Phys. 11, 1131 (1978).

[17] D. L. Andrews, Structured Light and Its Applications: An Introduction to Phase-Structured Beams and Nanoscale Optical Forces (Academic Press, Burlington, MA, 2011).

[18] H. Rubinsztein-Dunlop et al., J. Opt. 19, 013001 (2016).

[19] L. Allen, M. W. Beijersbergen, R. J. C. Spreeuw, and J. P. Woerdman, Phys. Rev. A 45, 8185 (1992).

[20] D. L. Andrews and M. Babiker, The Angular Momentum of Light (Cambridge University Press, Cambridge, UK, 2012).

[21] S. M. Barnett, M. Babiker, and M. J. Padgett, Optical Orbital Angular Momentum (The Royal Society, London, 2017).

[22] A. Zangwill, Modern Electrodynamics (Cambridge University Press, Cambridge, UK, 2013).

[23] U. D. Jentschura and V. G. Serbo, Phys. Rev. Lett. 106, 013001 (2011).

[24] R. P. Cameron and S. M. Barnett, Phys. Chem. Chem. Phys. 16, 25819 (2014).

[25] S. Stock, A. Surzhykov, S. Fritzsche, and D. Seipt, Phys. Rev. A 92, 013401 (2015).

[26] J. Li, J. J. Tu, and J. L. Birman, J. Phys. Chem. Solids 77, 117 (2015).

[27] J. A. Sherwin, Phys. Rev. A 95, 052101 (2017).

[28] A. A. Peshkov, A. V. Volotka, A. Surzhykov, and S. Fritzsche, Phys. Rev. A 97, 023802 (2018).

[29] D. McArthur, A. Yao, and F. Papoff, arXiv:1908.03439.
[30] A. A. Peshkov, S. Fritzsche, and A. Surzhykov, Phys. Scr. 94, 105402 (2019).

[31] H. Yu, J. Park, K. Lee, J. Yoon, K. Kim, S. Lee, and Y. Park, Curr. Appl. Phys. 15, 632 (2015).

[32] S. Rotter and S. Gigan, Rev. Mod. Phys. 89, 015005 (2017).

[33] C. T. Schmiegelow and F. Schmidt-Kaler, Eur. Phys. J. D 66, 157 (2012).

[34] V. E. Lembessis and M. Babiker, Phys. Rev. Lett. 110, 083002 (2013).

[35] M. G. Mandujano and J. A. Maytorena, Phys. Rev. A 88, 023811 (2013).

[36] A. Afanasev, C. E. Carlson, and A. Mukherjee, J. Opt. 18, 074013 (2016).

[37] M. Babiker, D. L. Andrews, and V. E. Lembessis, J. Opt. 21, 013001 (2019).

[38] D. L. Andrews, G. A. Jones, A. Salam, and R. G. Woolley, J. Chem. Phys. 148, 040901 (2018).

[39] L. D. Romero, D. L. Andrews, and M. Babiker, J. Opt. B: Quantum Semiclassical Opt. 4, S66 (2002).

[40] D. L. Andrews and K. A. Forbes, Opt. Lett. 43, 3249 (2018).

[41] D. S. Bradshaw and D. L. Andrews, Eur. J. Phys. 38, 034008 (2017).

[42] D. L. Andrews, in Complex Light Opt. Forces V (International Society for Optics and Photonics, SPIE, Bellingham, WA, 2011), p. 79500L.

[43] N. Zhao, X. Li, G. Li, and J. M. Kahn, Nat. Photonics 9, 822 (2015).

[44] W. B. Wang, R. Gozali, L. Shi, L. Lindwasser, and R. R. Alfano, Opt. Lett. 41, 2069 (2016).

[45] L. Gong, Q. Zhao, H. Zhang, X.-Y. Hu, K. Huang, J.-M. Yang, and Y.-M. Li, Light Sci. Appl. 8, 27 (2019).

[46] L. D. Barron, Molecular Light Scattering and Optical Activity (Cambridge University Press, Cambridge, UK, 2009).

[47] L. D. Barron and A. D. Buckingham, Chem. Phys. Lett. 492, 199 (2010).

[48] D. L. Andrews, J. Opt. 20, 033003 (2018).

[49] K. A. Forbes, Phys. Rev. Lett. 122, 103201 (2019).

[50] K. A. Forbes and D. L. Andrews, Phys. Rev. Res. 1, 033080 (2019).

[51] D. L. Andrews, L. D. Romero, and M. Babiker, Opt. Commun. 237, 133 (2004).

[52] K. A. Forbes and D. L. Andrews, Opt. Lett. 43, 435 (2018).

[53] K. A. Forbes and D. L. Andrews, Phys. Rev. A 99, 023837 (2019).

[54] R. P. Cameron and N. Mackinnon, Phys. Rev. A 98, 013814 (2018).

[55] B. Paroli, M. Siano, and M. A. C. Potenza, Opt. Express 27, 17550 (2019).

[56] Y. Shen, G. T. Campbell, B. Hage, H. Zou, B. C. Buchler, and P. K. Lam, J. Opt. 15, 044005 (2013). 
[57] A. S. Ostrovsky, C. Rickenstorff-Parrao, and V. Arrizón, Opt. Lett. 38, 534 (2013).

[58] S. Fu, T. Wang, and C. Gao, J. Opt. Soc. Am. A 33, 1836 (2016).

[59] Y. Chen, Z.-X. Fang, Y.-X. Ren, L. Gong, and R.-D. Lu, Appl. Opt. 54, 8030 (2015).
[60] D. L. Andrews and T. Thirunamachandran, J. Chem. Phys. 67, 5026 (1977).

[61] S. M. Barnett, J. Opt. B: Quantum Semiclassical Opt. 4, S7 (2001).

[62] K. Y. Bliokh, F. J. Rodríguez-Fortuño, F. Nori, and A. V. Zayats, Nat. Photonics 9, 796 (2015). 